\title{
Disparities in Diabetes Care
}

\author{
Authors: \\ Adrian Po Zhu Li, ${ }^{1}{ }^{*}$ Martin Brunel Whyte ${ }^{1,2}$ \\ 1. Department of Diabetes, King's College NHS Foundation Trust, London, UK \\ 2. Department of Clinical \& Experimental Medicine, University of Surrey, Guildford, UK \\ *Correspondence to m.b.whyte@surrey.ac.uk \\ Disclosure: $\quad$ The authors have declared no conflicts of interest. \\ Received: $\quad 04.09 .21$ \\ Accepted: $\quad 27.09 .21$ \\ Keywords: Diabetes mellitus, disparities, ethnicity, inequality, socioeconomic. \\ Citation: $\quad$ EMJ Diabet. 2021;9[1]:92-101.
}

\section{Abstract}

Disparities in the distribution of diabetes health have been reported by social class, age, gender, and ethnicity and may arise from an interplay of biological, clinical, and non-clinical factors. As well as being morally wrong, these differences in outcome will have a significant adverse effect on a nation's health. As a result, there have been international efforts to reduce inequalities, from the strategic organisation of healthcare to providers and patients themselves, with mixed effects. This article outlines the disparities in diabetes care and outcomes in different patient groups, and how the approach of integration of health and social care may help to overcome some of the adverse aspects of societal organisation that underpins disparities.

\section{INTRODUCTION}

Healthcare disparities and inequality are concepts that reflect aspects of differential healthcare access, disease and symptom management, and healthcare outcomes. Health inequalities can be defined as the "preventable, unfair and unjust differences in health status between groups, populations, or individuals that arise from the unequal distribution of social, environmental, and economic conditions within societies, which determine the risk of people getting ill, their ability to prevent sickness, or opportunities to take action and access treatment when ill health occurs." Historically, disparities purely referred to a difference of some kind but, in recent years, the term has come to be synonymous with unfairness and inequality.

Having a focus on improving inequalities, rather than on raising the average health of the nation,

is not just a question of fairness and social justice. Inequalities may be readily avoidable by governmental healthcare policy, as well as being economically advantageous to society. Globally, there is a large body of evidence documenting inequalities in access to healthcare and health outcomes in diabetes-related areas. $^{2-6}$ The distribution of health is determined by a wide variety of individual, community, and national factors. The Dahlgren and Whithead model (Figure 1) illustrates the contribution size of each layer to health, indicates the feasibility of changing specific factors, and the complementary action that would be required to influence linked factors in other layers. ${ }^{2}$

Inequalities in the distribution of health have been reported by social class, age, gender, and ethnicity. In all countries (whether low-, middle-, or high-income) there are wide disparities in the health status of different socioeconomic groups. ${ }^{7}$ Evaluating outcomes by 'ethnicity' is 


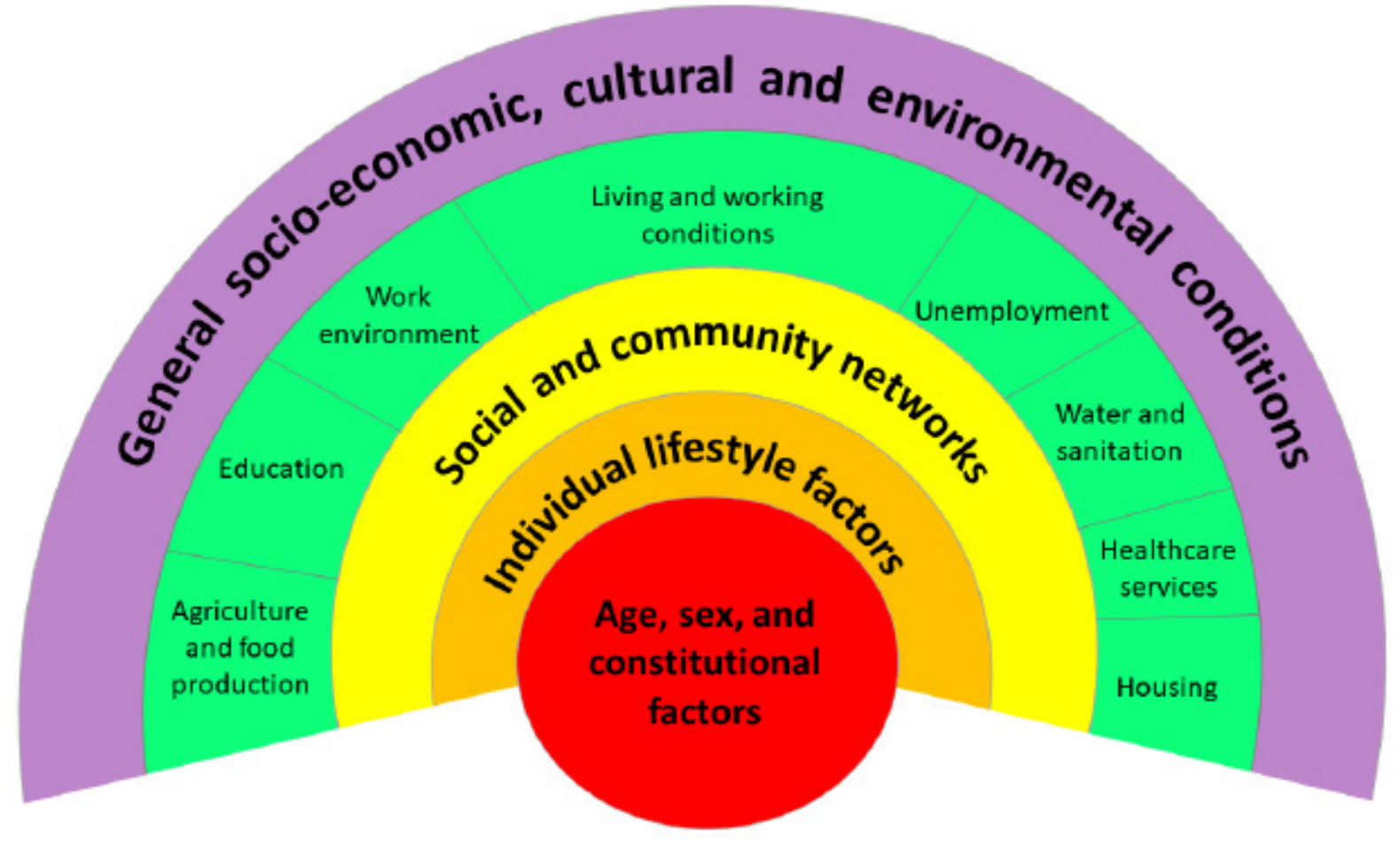

Figure 1: The Dahlgren and Whitehead model maps the relationship between the individual, their environment, and health.

more problematic in that the definition and interpretation of ethnicity is influenced by both historical value systems and the current social and political context. For example, reference to 'Asian' minority groups in USA (often south-east Asian diaspora) may differ to that of UK (often the Indian subcontinent).

Type 1 diabetes mellitus (T1DM) and Type 2 diabetes mellitus (T2DM) are both considered to be life-long conditions (with bariatric surgery and very low-calorie diets being notable exceptions leading to T2DM remission), whose aetiology has environmental and genetic contributions. From the outset, therefore, the impact on populations will be unequal. Diabetes is one of the most common chronic diseases and places a sizeable burden on patients, healthcare systems, and society. The International Diabetes Federation (IDF) estimate that nearly 700 million adults will be living with diabetes worldwide by $2045 .^{8}$ Diabetes is among the top 10 causes of adult mortality, and was estimated to have caused 4 million deaths globally in 2017.9 The burden of morbidity and mortality from diabetes is unequally shared, and these disparities in diabetes outcomes arise from a complex interplay of biological, clinical, and non-clinical factors. ${ }^{10}$ These factors will be explored in this article.

\section{SEARCH METHODOLOGY}

The authors searched English-language literature to identify all relevant studies in the last decade; from the year 2010 to the present date, regardless of publication status. They searched PubMed and Google Scholar databases, combining the terms 'diabetes' AND 'disparity' OR 'inequality' AND 'socio-economic' OR 'minority' OR 'ethnicity'. The authors have not covered gender disparities, which are extensively covered elsewhere." They applied backward and forward snowballing to identify further papers. An extensive list was developed, and a shortlist was created based on the limitations of the length of the narrative review and importance of the marker. The last search was performed in June 2021. 


\section{EPIDEMIOLOGY OF DIABETES DISPARITIES}

\section{Inequalities in Diabetes Incidence}

The incidence of T1DM increases towards the Northern and Southern poles of the world. Even within the UK, Northern areas of Scotland will have a higher incidence than in the South. Worldwide, disease onset of T1DM is associated with higher socioeconomic status (SES). In Europe, higher rates are reported in host White populations but increasing rates in second-generation migrants are reported.12 T2DM is predisposed by obesity, which is itself socially patterned, with higher rates in lower socioeconomic groups. In the USA, diabetes is $60 \%$ more common in Black Americans than in White Americans, ${ }^{13}$ and Indigenous American and Alaskan Native populations have diabetes prevalence twice that of the general population. ${ }^{14}$ In the Pacific, diabetes disproportionally affects Hawaiian and Pacific Islander populations, the latter group having much higher rates in New Zealand than the White population. ${ }^{15}$ Within Western European countries, the prevalence of T2DM is higher in ethnic minorities, particularly in those of South Asian, Middle Eastern, and North African origin. ${ }^{10}$ Ethnic minority groups are also likely to develop T2DM at a younger age (up to 12 years younger), compared with their White counterparts. ${ }^{10}$

\section{Disparities in Rates of Diabetes Complications and Control}

\section{Macrovascular}

In a recent meta-analysis of worldwide studies (from USA, Canada, UK, and New Zealand), greater all-cause mortality in diabetes is not seen in Black or Asian populations compared with White populations. However, there remains a significantly greater mortality in the Māori population than the White population in New Zealand. ${ }^{15}$ Behind this headline figure, there are differences in subtypes of cardiovascular disease. Cardiovascular disease is more prevalent in the Indigenous American and Alaskan Native populations than the non-Hispanic White population (14.7\% compared with $12.2 \%$, respectively).$^{14}$ Hispanic American participants have a lower risk of CVD than White participants (hazard ratio: 0.66 [95\% confidence interval: 0.53-0.81]). ${ }^{15}$ Black people with diabetes have an equivalent overall cardiovascular event rate to White people, but Black individuals tend to have an equal or lower risk of coronary heart disease..$^{15}$ This may relate to higher high-density lipoprotein cholesterol and lower triglyceride levels (unattributable to dietary difference) ${ }^{16}$ compared with White people. ${ }^{17}$ It is important to consider that rates can change with acculturation. ${ }^{10}$

The prevalence of diagnosed and undiagnosed hypertension among African American males (42.4\%) and females (44\%) $\geq 20$ years of age in the USA is higher than the expected base rate of approximately $30 \% .^{18}$ The origins of adult differences in hypertension begin early, with $13.8 \%$ prevalence of hypertension in African American youths versus $8.4 \%$ in the White subgroup and $10.4 \%$ in Hispanic populations. ${ }^{19}$ Likely as a consequence of this, Black people in the USA between 45- and 64-years-old have a 3-fold higher risk of stroke compared to the White community. ${ }^{20}$ Conversely, ethnic differences in risk of stroke have not been evident in the UK. ${ }^{21,22}$

\section{Microvascular}

\section{Retinopathy}

Predictors for diabetic retinopathy include older age (and younger age at diabetes diagnosis), male sex, Black and Asian race, socioeconomic deprivation, and occupation. ${ }^{23}$ In the USA, rates of retinopathy are higher among non-White ethnic groups, ${ }^{24}$ whereas in the UK, risk of retinopathy is equal in both Black and White populations ${ }^{25}$ and lower in the South Asian community. ${ }^{26}$ Adverse retinal outcomes including sight-threatening retinopathy in those with lower SES persist despite universal screening programmes. ${ }^{27}$ Underpinning this microvascular burden is greater prevalence of vascular risk factors. Patients living in deprived areas will less often achieve glycaemic control targets and tend to have higher blood pressure and worse lipid profile control. ${ }^{28}$

\section{Kidney disease}

It is consistently reported that ethnic minorities have a higher prevalence of diabetes and chronic kidney disease than White individuals. ${ }^{15,29}$ For example, in the USA, Hispanic individuals have a 2-fold and Black individuals a 3- to 4-fold 
greater risk compared to White individuals. ${ }^{15,30}$ In the UK, people of both South Asian and Black ethnic origin have 3- to 4-fold higher rates of acceptance onto renal replacement therapy than White individuals, ${ }^{31}$ which can only be partly explained by a higher prevalence of T2DM and hypertension (in the Black population).

\section{Neuropathy and foot care}

Despite a lower prevalence of clinical neuropathy in South Asian individuals compared to individuals of White or Black African or Caribbean ethnicity, in the UK people of an Asian background with T2DM appear at greater risk of painful diabetic neuropathy. ${ }^{32}$

There are international differences in the epidemiology of diabetic foot disease, which could be explained by the differences in economic viability and governmental infrastructures. However, there is also marked variation within countries. In the USA, the risk of foot ulceration and lower limb amputation tends to be greater in non-White people, ${ }^{33,34}$ but in the UK this is not the case. Compared with White Europeans, Black individuals of African or Caribbean or South Asian descent have been found to have a reduced risk of lower limb amputation. ${ }^{35,36}$ Socioeconomic disparities in diabetic foot care have been demonstrated, particularly in the USA, where there is a greater prevalence of lower-extremity amputations and peripheral vascular disease in lower-income regions and minority groups. ${ }^{5}$

\section{CONTRIBUTORS TO DISPARITIES IN OUTCOMES}

\section{Biological Factors for Diabetes and Its Complications}

Whether genetic factors contribute to ethnic inequalities in T2DM is unclear as there have been so few studies of ethnic minorities in Western countries. The interplay with the environment is not to be underestimated, as rates of T2DM are four times higher for those of Indian ancestry living in Western Europe compared to rates in the Indian subcontinent, and even higher for those of African descent. ${ }^{10}$ Differences in fat distribution between the visceral and subcutaneous depots between ethnicities will affect insulin resistance and partly contribute to the higher rates of diabetes in the South Asian and Black African ethnic groups. ${ }^{10}$ Higher post-prandial glucose, implicated in cardiovascular disease, has been reported in South Asians. ${ }^{37,38}$ There remains uncertainty as to whether the predominant pathophysiological mechanism in the development of T2DM differs according to ethnicity, particularly regarding $\beta$-cell secretory capacity. Genetic markers for T2DM appear to differ between racial groups, but it remains uncertain as to how much these account for disparity in diabetes prevalence. ${ }^{39}$ However, more data output for complication risk is needed. For instance, risk variants in the APOL1 gene on chromosome 22, initially discovered in the African American population, are associated with an increased risk of kidney disease. ${ }^{15}$

\section{Social Determinants of Health}

SES is a multifaceted formulation that includes educational, economic, and occupational status. Each contributes overlapping properties to health. An individual's highest attained level of education is generally reached in early adulthood. Thereafter, their health will be driven by living conditions, better healthcare, and lifestyle (Figure 2). ${ }^{40}$ Their income will dictate the built environment in which they live and their access to food and affordable healthcare. Their occupation may be associated with toxic environmental exposures and food availability.

People from lower SES may engage less with healthcare and have higher rates of nonattendance to appointments. Cumulatively, low SES can have the same adverse impact on health as smoking or low exercise levels. ${ }^{41}$ The effect of macroeconomic factors on diabetes was well illustrated by the economic recession of 2008. Living in towns with higher household incomes led to the achievement of significantly better performance in diabetes care indicators. ${ }^{42}$ More generally, there are no data as to whether changes in income, higher educational status, or different employment/ occupational status improves diabetes outcomes.

\section{Disparities in Quality of Healthcare and Access to Drugs and Technology}

Those at highest need are reported to be the least likely to receive healthcare, a phenomenon known as Hart's inverse care law (Figure 3). 
Potential confounders

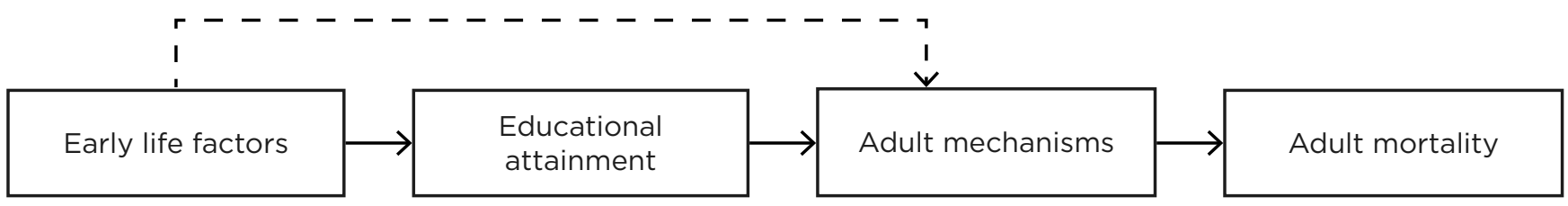

Parental education and income

Individual

endowments

(eg. genetics and

intelligence)

Childhood physical

and mental health

Childhood social

context
Valued information about and support for healthy lifestyles and healthcare

Access to good jobs and associated rewards in an information-based society

Access to valuable networks and relationships, perhaps increasingly so with technological advancements and ease of travel

Sophisticated cognitive skills, greater sense of control, and human agency

Figure 2: The relationship between educational attainment and adult mortality.

Reproduced with permission from Hayward et al. ${ }^{40}$

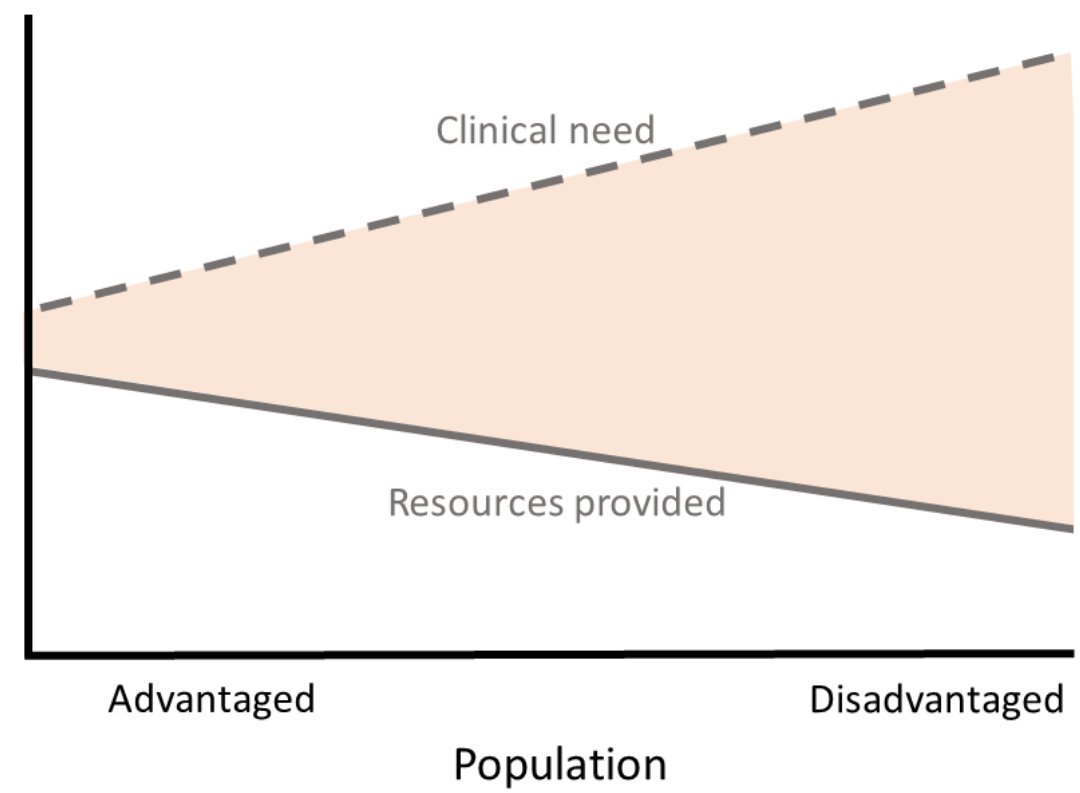

Less resources despite greater need in disadvantaged groups

Figure 3: Hart's inverse care law. 
In contrast, socially disadvantaged people in high-income countries may receive more healthcare, but it is of worse quality and insufficient quantity to meet their additional needs, known as the 'disproportionate care law'. In addition to the availability of healthcare services and the quality of the services offered, Goddard and Smith highlighted two other reasons for variations in access to healthcare: direct and/or indirect costs of healthcare, and the quality of information provided to all population groups. ${ }^{43}$ Groups who are considered hard to reach tend to access health services less frequently and suffer poorer health outcomes. Such people may include minority ethnic groups, the homeless, asylum seekers, the unemployed, the elderly, people with learning disabilities, and people with mental health or substance misuse problems. These individuals may ill-afford access to healthcare and/or be provided with unsuitable information.

In a 2016 publication, the IDF reported that access to diabetes drugs in developing countries was a particular concern. ${ }^{4}$ For instance, metformin is usually the first-line treatment for T2DM, but comprehensive government provision of metformin was limited to $10 \%$ of low-income countries versus $72 \%$ of high-income countries. ${ }^{4}$ Access to sulfonylureas and dipeptidyl peptidase 4 inhibitors fared worse, with none of the lowincome countries having government provision. Similar trends existed for insulin. Consequently, people from low-income countries can spend up to two-thirds of their disposable income to pay for insulin and essential consumables associated with insulin administration such as needles and glucometers, both of which were sparser than insulin. ${ }^{4}$

Disparities in prescribing trends also exist within developed countries. In the UK and Australia, SES is a key determinant of disparity in glycaemic control and for the prescription of newer therapies for T2DM. ${ }^{6}$ Ethnicity also leads to variable prescribing. In the UK, people of Asian ethnicity were $32 \%$ less likely to be prescribed sodium-glucose co-transporter-2 inhibitors and $63 \%$ less likely to be prescribed glucagon-like peptide-1 agonists. ${ }^{28}$ One factor that may affect prescribing is the reliance on $\mathrm{BMI}$ in therapeutic decision trees. According to the World Health Organization (WHO) classifications, a BMI of $30 \mathrm{~kg} / \mathrm{m}^{2}$ is considered obese. However, this threshold is not suitable for people with Black, Asian, and minority ethnic backgrounds, who have the same risk for T2DM and at a BMl of $27 \mathrm{~kg} / \mathrm{m}^{2}$ and $22 \mathrm{~kg} / \mathrm{m}^{2}$, respectively, as their White counterparts would with a BMI of $30 \mathrm{~kg} / \mathrm{m}^{2}{ }^{10}$ As prescribing of glucagon-like peptide-1 agonists is often based on BMI, this will have the effect of discriminating against the non-White population.

Many diabetes drugs require dose reduction or cessation with kidney impairment. Calculations for the estimated glomerular filtration rate adjust for ethnicity, which may itself entrench disparity. A recent study showed that removal of race adjustment may increase diagnoses of chronic kidney disease among Black adults and, thereby, enhance access to specialist care. However, such a change may also prompt drug contraindications or dose reductions for individuals who are reclassified to advanced stages of chronic kidney disease. ${ }^{44}$ Consequently, people from African Caribbean backgrounds may have an underestimate of the estimated glomerular filtration rate and, thus, may be wrongly denied access to therapy such as sodium-glucose co-transporter-2 inhibitors. ${ }^{28,44}$

Recent advances for T1DM diabetes include continuous glucose monitoring devices and insulin pump therapy. Significant variations exist in access to such technologies, which are associated with suboptimal glycaemic control. Increasing age is negatively correlated with computer literacy, hence the increase in diabetes technology use has primarily benefited young and middle-aged individuals. ${ }^{45}$ Regional variation in provision also affects use. There is a ten-fold variation in insulin pump use across specialist centres in the UK. ${ }^{46}$ People from lower SES groups tend to have reduced computer use and computer experience, although this has lessened in recent years. ${ }^{47}$ This digital divide restricts access to technologies where home-based uploading of data is required. The uptake of virtual consultations for diabetes during the COVID-19 pandemic may exacerbate this disparity in computer-access and literacy. 


\section{INTERVENTIONS TO REDUCE DISPARITIES}

Interventions to reduce disparity can occur at various levels, as depicted on the Dahlgren and Whitehead model (Figure 1), from the individual to wider-scale healthcare and societal organisation.

\section{Healthcare Organisation}

The principal determinants of inequalities in health are national socioeconomic factors and the physical and social environment. ${ }^{48}$ The complexity of the causes of inequalities in health means that multifaceted and, therefore, multi-sectoral action is required to tackle the problem. A key component is the organisation of the healthcare system and interventions here have the potential to effect significant changes in healthcare processes and health outcomes. Provision of universal coverage is a necessary, but not sufficient, requirement for achieving equity in healthcare. In diabetes, supporting evidence comes from populationbased studies: having health insurance is the strongest predictor of whether individuals have access to diabetes screenings and care. ${ }^{49}$

National retinopathy screening programmes can help to equalise access to ophthalmic support and ensure people of all ages have regular riskstratification to prevent worsening retinopathy and blindness. A difficulty in determining the effect of screening programmes or preventive therapy is the 'healthy user effect'. This arises as healthier patients are more likely to attend screening programmes and/or more likely to request prescriptions for preventive therapies. Non-attendance to screening appointments exhibits a bimodal distribution, with higher rates in those between 16-30 years of age and a second peak in patients over the age of 90 years. ${ }^{30}$ Automated appointment reminders aim to improve this but fall foul of disparities in computer literacy.

Pay-for-performance strategies, which financially reward the achievement of targets such as blood pressure and cholesterol, were introduced to strengthen primary care in UK but have been found to fall short of addressing the disparities in diabetes management between minority ethnicity groups. ${ }^{50}$

\section{Approaches for Medical and Social Care Integration}

Given that diabetes is predominantly managed in the community, successful interventions should be based in the community setting. Two main types of intervention to address social determinants of health are compensatory interventions, which provide support to enable individuals to fill gaps and access otherwise inaccessible or unavailable resources; and root cause interventions, which are designed to change underlying structures or systems, rather than compensate for them. ${ }^{51}$

An example of the former is the National Diabetes Prevention Plan in the USA and the National Health Service Diabetes Prevention Programme (NHS DPP) in the UK. Each supports those at high risk of T2DM to reduce their risk via a supported lifestyle intervention to achieve a healthy weight, improve nutrition, and increase physical activity. The standardised training of facilitators and certification serves to reduce disparity in quality between centres. A root cause approach to diabetes prevention may instead include components such as residential environment planning, allowing for walking and cycling, or the restriction of unhealthy businesses (e.g., fast foods) in lowincome areas. ${ }^{52}$

\section{Individual Healthcare Providers}

Most interventions to reduce diabetes disparities by SES have been conducted in industrialised countries. ${ }^{53}$ Positive interventions include cultural tailoring of the intervention; community educators or lay people leading the intervention; one-on-one interventions, incorporating treatment algorithms; and high-intensity interventions (at least 10 appointments) delivered over a long duration ( $\geq 6$ months). Less useful interventions were didactic teaching or interventions focused only on diabetes knowledge. ${ }^{53}$ One possible mediator of ethnicity on health disparity is the presence of a language barrier. Interpreter services in the USA have led to a greater frequency of health visits and completed prescriptions ${ }^{54}$ and, in the UK, patients with language barriers seen at language-concordant providers in primary care have reduced diabetes-related hospital admissions. ${ }^{55}$ 


\section{Patients}

Structured education and self-management are considered vitally important for diabetes care and yet self-deterministic interventions may widen inequalities, as disadvantaged groups are less likely to participate. ${ }^{56}$ Approaches that address financial burden as well as work and environment-related factors are essential for enhancing diabetes self-management.

Individual behaviours relating to food choice, inactivity, smoking, and alcohol need to be addressed. Smoking is significantly more common in socioeconomically deprived areas. Although rates are declining, this has been slower in disadvantaged groups. ${ }^{57}$ Differences in alcohol-related harm exist across Europe, where consumption of alcohol is the highest per capita in the world. ${ }^{58}$ In general, alcoholrelated harm is greater in people from lower SES groups, even if consumption is equal to more affluent counterparts. ${ }^{58}$ Education may paradoxically worsen inequalities of alcoholism, whereas measures limiting the availability of alcohol, including price rises and licence restrictions, has a disproportionate effect on lower SES groups. ${ }^{58}$

\section{CONCLUSIONS}

Disparities in diabetes care continue to exist between and within nations. These disparities lead to higher-risk groups having less access to optimal treatments for both T1DM and T2DM. They also contribute to the variation in microvascular and macrovascular complication rates. It is important, morally and for societal health at large, that these inequalities are addressed. Differences in morbidity between ethnic minority populations in North America and the UK are unlikely to be attributed to genetic differences but, rather, they infer differences in healthcare systems and healthcare access. Strategies to reduce health inequalities must be based on a societal healthcare policy. The implementation of strategies will need to be intersectoral and multidisciplinary. It is vital that interventions must be adequately funded and comprise of tailored interventions that are culturally sensitive, local to the individual with diabetes, and not didactic in nature.

\section{References}

1. NHS England. Reducing health inequalities resources, Available at: https://www.england.nhs.uk/about/ equality/equality-hub/resources/. Last accessed: 29 August 2021.

2. Health Knowledge. Inequalities in health (e.g. by region, ethnicity, socioeconomic position or gender) and in access to health care, including their causes. 2020. Available at: https://www.healthknowledge.org. uk/public-health-textbook/medicalsociology-policy-economics/4cequality-equity-policy/inequalitiesdistribution. Last accessed: 29 August 2021.

3. International Diabetes Federation (IDF). Annual report 2016. 2017. Available at: https://idf.org/ouractivities/advocacy-awareness/ resources-and-tools/123-idf-annualreport-2016.html. Last accessed: 29 August 2021

4. International Diabetes Federation (IDF). Access to medicines and supplies for people with diabetes. 2020. https://idf.org/our-activities/ care-prevention/access-to-medicine. html. Last accessed: 29 August 2021.

5. Mizelle Jr RM. Diabetes, race, and amputations. Lancet. 2021:397(10281):1256-7.

6. Morton $\mathrm{Jl}$ et al. The association of socioeconomic disadvantage and remoteness with receipt of type 2 diabetes medications in Australia: a nationwide registry study. Diabetologia. 2021;64(2):349-60

7. World Health Organisation (WHO) Health inequities and their causes. 2018. Available at: https://www.who int/news-room/facts-in-pictures/ detail/health-inequities-and-theircauses. Last accessed: 29 August 2021.

8. Saeedi P et al. Global and regiona diabetes prevalence estimates for 2019 and projections for 2030 and 2045: Results from the International Diabetes Federation Diabetes Atlas, $9^{\text {th }}$ edition. Diabetes Res Clin Pract. 2019;157:107843.

9. International Diabetes Federation (IDF). IDF diabetes atlas $8^{\text {th }}$ edition. 2017. Available at: https:// diabetesatlas.org/upload/resources/ previous/files/8/IDF_DA 8e-EN-final. pdf. Last accessed: 29 August 2021.

10. Goff LM. Ethnicity and type 2 diabetes in the UK. Diabet Med. 2019;36(8):927-38

11. The Lancet Diabetes Endocrinology Sex disparities in diabetes: bridging the gap. Lancet Diabetes Endocrinol. 2017;5(11):839.

12. Hussen $\mathrm{HI}$ et al. The trends and the risk of type 1 diabetes over the past 40 years: an analysis by birth cohorts and by parental migration background in Sweden. BMJ Open. 2013;3(10):e003418.

13. Centers for Disease Control and Prevention (CDC). Tables of summary health statistics. Available at: https:// www.cdc.gov/nchs/nhis/shs/tables. htm. Last accessed: 19 Sept 2021.

14. Poudel $A$ et al. Diabetes and associated cardiovascular complications in American Indians/ Alaskan Natives: a review of risks and prevention strategies. J Diabetes Res. 2018:2018:2742565.

15. Ezzatvar $Y$ et al. Racial differences in all-cause mortality and future complications among people with diabetes: a systematic review and meta-analysis of data from more than 2.4 million individuals. Diabetologia. 
2021.

16. Lindquist $\mathrm{CH}$ et al. Role of dietary factors in ethnic differences in early risk of cardiovascular disease and type 2 diabetes. Am J Clin Nutr. 2000;71(3):725-32.

17. Osei K, Gaillard T. Disparities in cardiovascular disease and type 2 diabetes risk factors in blacks and whites: dissecting racial paradox of metabolic syndrome. Front Endocrinol (Lausanne). 2017;8:204.

18. Carnethon MR et al. Cardiovascular health in African Americans: a scientific statement from the American Heart Association. Circulation. 2017;136(21):e393-e423.

19. National High Blood Pressure Education Program Working Group on High Blood Pressure in Children and Adolescents. The fourth report on the diagnosis, evaluation, and treatment of high blood pressure in children and adolescents. Pediatrics. 2004;114(2 Suppl 4th Report):555-76.

20. Howard VJ et al. Sex and race differences in the association of incident ischemic stroke with risk factors. JAMA Neurol. 2019;76(2):17986.

21. Owusu Adjah ES et al. Prevalence and incidence of complications at diagnosis of T2DM and during followup by BMI and ethnicity: a matched case-control analysis. Cardiovasc Diabetol. 2018;17(1):70.

22. Tillin $\mathrm{T}$ et al. The relationship between metabolic risk factors and incident cardiovascular disease in Europeans, South Asians, and African Caribbeans: SABRE (Southall and Brent Revisited) -- a prospective population-based study. J Am Coll Cardiol. 2013;61(17):1777-86.

23. Haider $\mathrm{S}$ et al. Prognostic prediction models for diabetic retinopathy progression: a systematic review. Eye (Lond). 2019;33(5):702-13.

24. Lanting LC et al. Ethnic differences in mortality, end-stage complications, and quality of care among diabetic patients: a review. Diabetes Care. 2005;28(9):2280-8.

25. Chaturvedi $\mathrm{N}$ et al. Differences in mortality and morbidity in African Caribbean and European people with non-insulin dependent diabetes mellitus: results of 20 year follow up of a London cohort of a multinational study. BMJ. 1996;313(7061):848-52.

26. Mather HM et al. Comparison of prevalence and risk factors for microalbuminuria in South Asians and Europeans with type 2 diabetes mellitus. Diabet Med. 1998;15(8):672-7.

27. Nugawela MD et al. Ethnic disparities in the development of sightthreatening diabetic retinopathy in a UK multi-ethnic population with diabetes: an observational cohort study. J Pers Med. 2021;11(8):740.
28. Whyte $M B$ et al. Disparities in glycaemic control, monitoring, and treatment of type 2 diabetes in England: a retrospective cohort analysis. PLoS Med. 2019;16(10):e1002942

29. Spanakis EK, Golden SH. Race/ethnic difference in diabetes and diabetic complications. Curr Diab Rep. 2013:13(6):814-23.

30. Ellis DA et al. Demographic and practice factors predicting repeated non-attendance in primary care: a national retrospective cohort analysis. Lancet Public Health. 2017;2(12):e551-

31. Mathur R et al. Ethnic differences in the progression of chronic kidney disease and risk of death in a UK diabetic population: an observational cohort study. BMJ Open. 2018;8(3):e020145.

32. Abbott CA et al. Prevalence and characteristics of painful diabetic neuropathy in a large communitybased diabetic population in the U.K. Diabetes Care. 2011;34(10):2220-4.

33. Sharpe JE. Eliminating the health disparity of the diabetic foot: a continuing public health crisis for African Americans and Latinos. Int $J$ Diabetes Clin Res. 2020;7:120.

34. Tan TW et al. Disparities in outcomes of patients admitted with diabetic foot infections. PLoS One. 2019;14(2):e0211481.

35. Gujral JS et al. Ethnic differences in the incidence of lower extremity amputation secondary to diabetes mellitus. Diabet Med. 1993;10(3):271-4

36. Leggetter S et al. Ethnicity and risk of diabetes-related lower extremity amputation: a population-based, case-control study of African Caribbeans and Europeans in the United Kingdom. Arch Intern Med. 2002;162(1):73-8.

37. Dickinson S et al. Postprandial hyperglycemia and insulin sensitivity differ among lean young adults of different ethnicities. J Nutr. 2002;132(9):2574-9.

38. Ludwig DS et al. A physiological basis for disparities in diabetes and heart disease risk among racial and ethnic groups. J Nutr. 2002;132(9):2492-3.

39. Staiano AE et al. Uncovering physiological mechanisms for health disparities in type 2 diabetes. Ethn Dis. 2015;25(1):31-7.

40. Hayward MD et al. Trends and group differences in the association between educational attainment and U.S. adult mortality: implications for understanding education's causal influence. Soc Sci Med. 2015;127:8-18.

41. Saydah SH et al. Socioeconomic status and mortality: contribution of health care access and psychological distress among U.S. adults with diagnosed diabetes. Diabetes Care.
2013;36(1):49-55

42. Casanova $L$ et al. [Trends in social inequities in diabetes care in the Provence-Alpes-Côte-d'Azur region of France between 2008 and 2011]. Rev Epidemiol Sante Publique. 2017;65(1):29-40. (In French).

43. Goddard M, Smith P. Equity of access to health care services: theory and evidence from the UK. Soc Sci Med. 2001;53(9):1149-62.

44. Diao JA et al. Clinical implications of removing race from estimates of kidney function. JAMA. 2021;325(2):184-6.

45. Toschi E, Munshi MN. Benefits and challenges of diabetes technology use in older adults. Endocrinol Metab Clin North Am. 2020;49(1):57-67.

46. Barnard-Kelly KD, Chernavvsky D. Social inequality and diabetes: a commentary. Diabetes Ther. 2020;11(4):803-11.

47. OECD. Inequalities in digital proficiency: bridging the divide. Available at: https://www.oecd-ilibrary.org/ docserver/9789264239555-8-en.pd f?expires $=1630309414 \& i d=i d \& a c c n a$ me=guest $\&$ checksum $=$ A1D 5052 DO 3 3E65981A81502C8C2DE658. Last accessed: 29 August 2021.

48. Crombie IK et al.; World Health Organisation (WHO). Closing the health inequalities gap: an international perspective. 2005. Available at: https://apps.who.int/iris/ handle/10665/107680. Last accessed: 29 August 2021.

49. Kazemian $P$ et al. Evaluation of the cascade of diabetes care in the United States, 2005-2016. JAMA Intern Med. 2019;179(10):1376-85

50. Millett $\mathrm{C}$ et al. Ethnic disparities in diabetes management and payfor-performance in the UK: the Wandsworth Prospective Diabetes Study. PLoS Med. 2007;4(6):e191.

51. Thornton PL et al. New research directions on disparities in obesity and type 2 diabetes. Ann N Y Acad Sci. 2020;1461(1):5-24.

52. Haire-Joshu D, Hill-Briggs F. The next generation of diabetes translation: a path to health equity. Annu Rev Public Health. 2019;40:391-410.

53. Glazier $\mathrm{RH}$ et al. A systematic review of interventions to improve diabetes care in socially disadvantaged populations. Diabetes Care. 2006;29(7):1675-88.

54. Jacobs EA et al. Overcoming language barriers in health care: costs and benefits of interpreter services. Am J Public Health. 2004;94(5):8669.

55. Hacker $\mathrm{K}$ et al. Exploring the impact of language services on utilization and clinical outcomes for diabetics. PLoS One 2012;7(6):e38507.

56. Gillett $M$ et al. Non-pharmacological 
interventions to reduce the risk of diabetes in people with impaired glucose regulation: a systematic review and economic evaluation. Health Technol Assess. 2012;16(33):1236.

57. National Centre for Smoking
Cessation and Training (NCSCT) Stop smoking services and health inequalities. 2013. Available at: https://www.ncsct.co.uk/usr/pub/ NCSCT_briefing_effect_of_SSS_ on_health_inequalities.pdf. Last accessed: 29 August 2021.
58. World Health Organization (WHO) Alcohol and inequities: guidance for addressing inequities in alcoholrelated harm. 2014. Available at: https://www.euro.who.int/_data/ assets/pdf file/0003/247629/ Alcohol-and-Inequities.pdf. Last accessed: 29 August 2021. 